\title{
Towards a Multi-camera Mouse-replacement Interface
}

\author{
John Magee, Zheng Wu, Harshith Chennamaneni, Samuel Epstein \\ Diane H. Theriault and Margrit Betke \\ Computer Science Department, Boston University \\ 111 Cummington St, Boston, MA 02215 U.S.A.
}

\begin{abstract}
We present our efforts towards a multi-camera mouse-replacement system for computer users with severe motion impairments. We have worked with individuals with cerebral palsy or multiple sclerosis who use a publiclyavailable interface that tracks the user's head movements with a single video camera and translates them into mouse pointer coordinates on the screen. To address the problem that the interface can lose track of the user's facial feature due to occlusion or spastic movements, we started to develop a multi-camera interface. Our multi-camera capture system can record synchronized images from multiple cameras and automatically analyze the camera arrangement. We recorded $15 \mathrm{sub}-$ jects while they were conducting a hands-free interaction experiment. We reconstructed via stereoscopy the three-dimensional movement trajectories of various facial features. Our analysis shows that single-camera interfaces based on twodimensional feature tracking neglect to take into account the substantial feature movement in the third dimension.
\end{abstract}

\section{Introduction}

Camera-based human-computer interaction via analysis of head movement has been studied for many years (e.g. [1-3]). Early work has typically focused on single-camera interfaces for entertainment, control of electronic devices (e.g., the remote control of a TV), and support of automated speech analysis. Camera-based human-computer interaction systems that serve as assistive communication tools have had an enormous impact on the lives of individuals with severe motion impairments [4, 5]. These systems function as mouse-replacement software that allow users to control a computer mouse pointer with head movements. The movements of the user's head [6], nose $[4,5,7]$ or other features [4] are converted into movements of the mouse pointer on the screen. To mimic the functionality of a left mouse click, a mouse replacement system typically evaluates the length of time that the pointer dwells over an icon, button, or menu item (or its surrounding region) and then issues a selection command.

Currently available video-based mouse-replacement systems for people with severe disabilities process the input video captured by a single camera. We propose a multicamera approach to alleviate the problems with track failures that such systems encounter in practice. Track failures occur when facial features become occluded during tracking, for example, when extreme head rotations result in self-occlusion of the feature. Track failures also occur due to involuntary rapid movements of users with spastic cerebral palsy. In addition, multiple camera systems may be able to use information 
about the user's motion in three dimensions to provide better control of the mouse pointer.

Data from more than one camera allows for use of a confidence measure computed from detecting and tracking the objects in different images and evaluating if they are consistent [8]. This may alleviate the problem of feature loss due to occlusion. For example, when the feature moves out of the field of vision of one of the cameras (the left nostril is occluded because the user turns left), the tracking of the feature is continued in another camera's field of view. We propose a camera placement that ensures that the fields of views of the cameras partially overlap. With this setup, if the feature is lost in one camera view, the tracker can use another camera view to continue tracking.

To facilitate research on multi-camera assistive technology, we developed a system that allows processing of images that are captured simultaneously from multiple cameras. Our Multi-Camera Capture (MCC) system provides the general framework for working with $n$ cameras and has been tested using up to four cameras simultaneously. The scope of the current project is limited to the following contributions:

1. We provide a software system that enables images to be recorded from multiple cameras.

2. We created a new database of videos of 15 subjects that are simultaneously recorded from three camera views while they were performing an interaction task.

3. We present a preliminary stereoscopic analysis of the three-dimensional trajectories of facial features during interaction experiments.

Our system uses inexpensive webcams, arranged on a desk in a typical human-computer interaction environment (Fig. 1), in contrast to expensive calibrated multi-camera capture systems in controlled laboratory environments. There is a need in the computer vision community to create data sets with which $3 \mathrm{D}$ face tracking systems can be evaluated [9]. This applies both to systems that use multiple cameras [9-12] or a single camera [13]. We hope that other computer-vision research groups will make use of our capture system and data, and help move forward the research on reliable communication systems for people with disabilities.

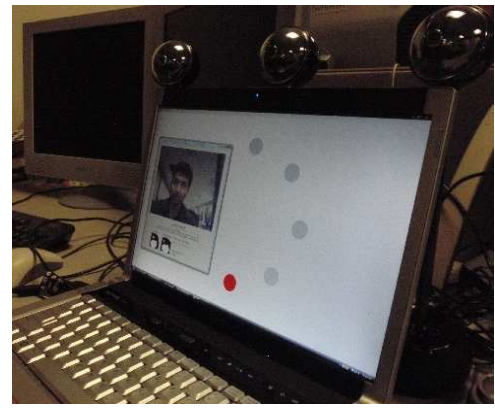

Fig. 1. A setup of the Multi Camera Capture system with three cameras. The cameras are the three silver-colored spheres on top of the laptop display. 


\section{Methods}

Our system divided into three modules, a multi-camera capturing module that provides temporal calibration, a module that evaluates the geometric relationship between the cameras and provides spatial calibration, and a third module that provides stereoscopic reconstruction of three-dimensional (3D) coordinates.

The first module, the Multi Camera Capture (MCC) program, records images from the cameras simultaneously and stores them. The second module performs camera calibration, that is to recover each camera's focal length, principal point, radial distortion coefficients (the "intrinsic parameters," which make up the camera matrix $K$ ) and spatial relationship between objects and cameras (the "extrinsic parameters" of rotation $R$ and translation $\mathbf{t}$ ). To estimate the intrinsic parameters, we use a planar calibration object chessboard with known physical size (Fig. 2). The use of a planar object allows us to focus on just two coordinates of each three-dimensional object point and determine the homography $H$, which maps points $(X, Y, 1)^{T}$ on the object plane to points $(x, y, 1)^{T}$ on the image plane, i.e., $(x, y, 1)^{T}=H \cdot(X, Y, 1)^{T}$. We are interested in estimating $H$ because it encodes the camera matrix $K$, the rotation matrix $R$, and the translation vector $\mathbf{t}$ :

$$
H=s K\left[r_{1} r_{2} \mathbf{t}\right],
$$

where $s$ is a scale factor and $r_{1}$ and $r_{2}$ are first two columns of the rotation matrix $R$. With a sufficient number of pairs of points on the chessboard and their corresponding image points, the matrix $H$ can be estimated using Singular Value Decomposition (SVD). With several images of the chessboard, oriented differently towards the camera, we can estimate multiple homography matrices $H_{i}$ for the same camera matrix $K$. The fact that vectors $r_{1}$ and $r_{2}$ are orthonormal provides additional constraints. Matrix $K$ is then estimated by solving a system of linear equations [14].

The intrinsic parameters of each camera, given by matrix $K$, only need to be estimated once and can then be used for stereoscopic reconstruction by our multi-camera capture system. To estimate the extrinsic parameters, we reuse the chessboard images to estimate the "fundamental matrix" that relates the coordinates of a feature in the image of one camera to the coordinates of the feature in the corresponding image of another camera. The program automatically estimates the positions of the corresponding points of pattern corners in each camera view. In particular, given the two-dimensional coordinates $q_{i}=\left(x_{i}, y_{i}\right)^{T}$ of a point in the $i$ th camera view and the two-dimensional coordinates $q_{j}=\left(x_{j}, y_{j}\right)^{T}$ of a corresponding point in the $j$ th camera view, the fundamental matrix relates the two via the equation

$$
q_{i}^{T} F_{i j} q_{j}=0
$$

The entries of the matrix $F_{i j}$ can be estimated using the 8-point algorithm [15], whose name comes from the number of corresponding point pairs $\left(q_{i}, q_{j}\right)$ that are used as input. Matrix $F_{i j}$ can also be estimated by using significantly more point pairs, incorporating the 8-point algorithm into a RANSAC framework [16]. It labels point pairs that do not agree with the most probable result as outliers. These point pairs may have been incorrectly identified as corresponding points. RANSAC is a non-deterministic algorithm that iterates through the possibilities of matrix entries until a desired level of 
accuracy is achieved. The desired level of accuracy is specified as a probability that the computed matrix is the most likely among all possible matrices. The set of point pairs used as input to the RANSAC algorithm may yield a number of all possible subsets of 8 corresponding points that is so large (e.g., order of millions) that it is computationally expensive to compute all matrices exhaustively.

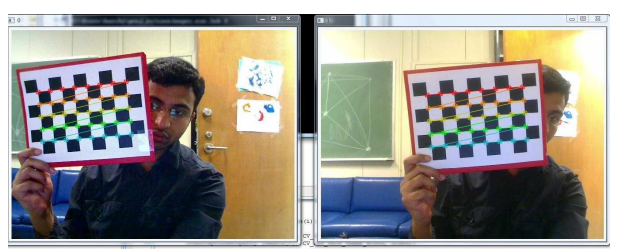

Fig. 2. A screenshot of the Multi Camera Capture system while it is used to record images simultaneously from two cameras in an experiment. In the field of view of the two cameras is a board with a checker pattern, which is used for spatial calibration of the cameras.

Our spatial calibration module calls the RANSAC method on 40 point pairs and uses an OpenCV function [14] to estimate the entries of the fundamental matrix. Our method uses a 99\%-probability level as the desired accuracy threshold. If this threshold yields a number of outlier pairs that exceeds 12 (i.e., $12 / 40 \approx 30 \%$ of the pairs), the points collected for stereo calibration are deemed to be insufficiently accurate, and a new calibration process is run.

Once the fundamental matrix $F_{i j}$ is determined, we can estimate the "essential matrix" $E_{i j}$ that encodes the absolute position and orientation of the two cameras:

$$
E_{i j}=K_{j}^{T} F_{i j} K_{i}
$$

To obtain the coordinates of corresponding points in three-dimensional world coordinates, we need to estimate the projection matrix $P=K[R \mid \mathbf{t}]$ of each camera. We assign the origin of the world-coordinate system to the center of projection of our first camera, which means that $P_{1}=K_{1}[I \mid \mathbf{0}]$. The projection matrices $P_{j}$ of the other cameras can then be constructed through SVD factorization of the essential matrices $E_{1 j}$ [15]. The projection matrices are stored by our calibration module and used for 3D reconstruction through triangulation by the third module of our system.

Our program relies on the known calibration device, the chessboard, for the calibration procedure. As an alternative, we have tracked distinctive features such as a human eye through several frames of each camera and used feature correspondences to perform self-calibration [15], that is, to estimate both intrinsic and extrinsic parameters for all cameras at the same time. The unknown scale can be estimated by measuring the physical distance between tracked features, e.g., the distance between a person's eyes.

\section{Experiments and Results}

We used a three-camera version of our multi-camera interaction system to record 15 subjects while they were conducting a human-computer interaction experiment. The 


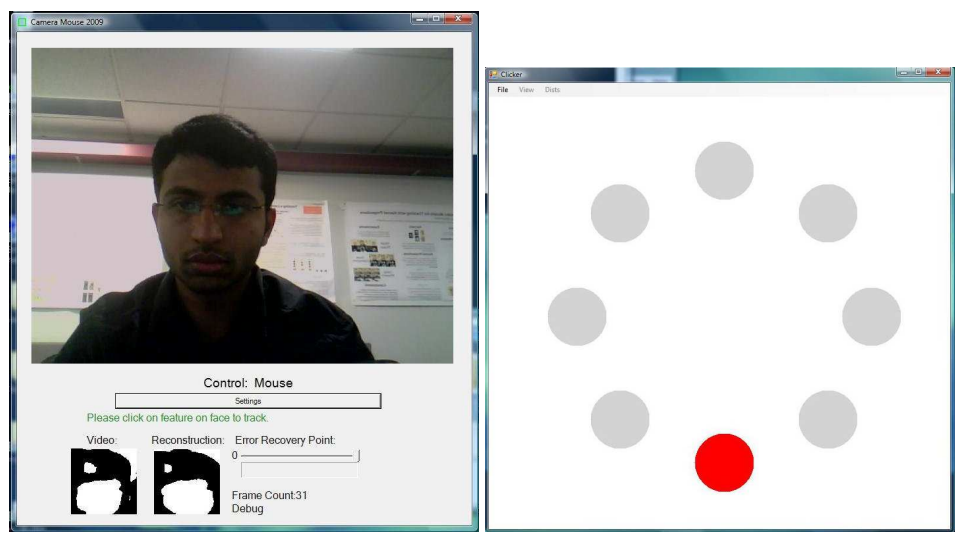

Fig. 3. Left: A screenshot of the Camera Mouse interface used in the experiments. Right: A screenshot of the ClickTester program. The user was instructed to move the mouse pointer into the target circle, highlighted in red, with head movements that were detected by the Camera Mouse. The ClickTester program presented a sequence of target circles to the user that was designed so that significant mouse pointer movements and changes in movement directions were required.

group of subjects included 8 men and 7 women. Most subjects had dark hair. Two subjects wore glasses. For each subject, we recorded three image sequences using three Logitech Orbit MP cameras (Fig. 1). The cameras were not radiometrically calibrated and each used automatic gain control. The recordings were synchronized by our temporal calibration module so that temporally corresponding images were identifiable.

Prior to the hands-free human-computer interaction experiment, the fundamental matrices for all pairs of cameras were estimated and stored using our spatial calibration module. We numbered the cameras from left to right starting with 0 . Our system then provided an estimate of the fundamental matrices $F_{01}, F_{12}$ and $F_{02}$ that relate the image coordinates of cameras 0 and 1,1 and 2 , and 0 and 2 , respectively. The spatial calibration module was executed before every subject test was performed in order to ensure spatial calibration via the three fundamental matrices. The camera positions were not disturbed during the recording.

We used the publicly-available assistive technology "Camera Mouse" [4, 17] (Fig. 3 left), which is a single-camera mouse-replacement system for people with severe motion impairments. We initialized the Camera Mouse using the standard mouse by selecting a facial feature (eyebrow corner) to track. The region around the corner of an eyebrow contains significant brightness changes, which makes it a reliable feature to track.

Our system recorded three image streams while the test subjects were moving their heads significantly (Fig. 4). We developed test software, called "Click Tester," that provided a movement protocol and ensured that all subjects were recorded with various head positions and orientations.

The ClickTester software displays eight circles on the screen, one of which is highlighted (see Fig. 3, right). In our experiments, the subject was asked to move the mouse pointer onto the highlighted circle using the Camera Mouse. When the subject had 


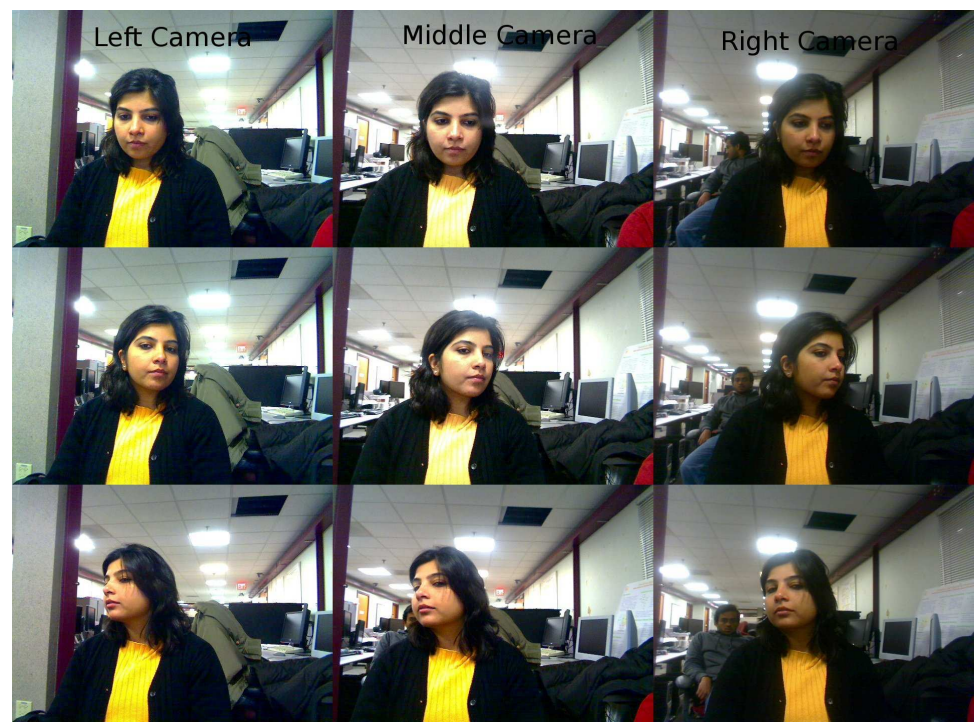

Fig. 4. Images collected at different points in time during the human-computer interaction experiment. Each row shows the three simultaneously-recorded frames as captured by the left, center, and right cameras. The subject's head orientation differs significantly from row to row and the subject's left eye is occluded in the right camera view in the second row. Similarly, the subject's right eye is occluded in the left camera view in the third row. The lack of radiometrical calibration of the cameras and the use of separate automated gain controls resulted in images with different intensity levels. This is particularly noticeable in the images recorded by the right camera, which are darker than the images recorded by the left and center cameras.

moved the mouse pointer to the highlighted circle, a new circle was highlighted. The subject was asked to repeat the process until all circles were visited. The software was designed as a means to simulate a realistic use of a camera-based mouse-replacement system that involved significant head motions. It also records the trajectory of the mouse pointer for further analysis.

Among the head motions that the subjects performed was a gesture in which the subjects moved their heads first upwards and then diagonally to the lower left. One of the subjects performing this gesture is shown in Fig. 5. His gesture lasted about three seconds, which corresponded to 17 frames. Ten of these 17 frames, as recorded by each of the three cameras, are shown in Fig. 5. We reconstructed the 3D positions of the outer corners of the subject's eyes and his nose tip during the head gesture. The 3D trajectories of the three features are shown in Fig. 6. We placed the 3D scene coordinate system so that its $x$ and $y$ axes were aligned with the image plane of the left camera and the $z$ axis was normal to and pointed away from the image plane. The origin of the 3D scene coordinate system was placed at the location of the nose tip in the first frame of the left camera view.

During the gesture, the features moved on average $18.5 \mathrm{~cm}$ in the $3 \mathrm{D}$ scene. It is noteworthy that, during the head gesture, the features moved $4.6 \mathrm{~cm}$ in the direction away from the left camera when the user directed the mouse pointer to the top 
of the computer screen. The features then moved back $3.0 \mathrm{~cm}$ towards the left camera when the user moved the mouse pointer to a region near the left corner of the screen. This component of a user's head movement cannot be taken into account by a singlecamera mouse-replacement interface that is based on two-dimensional tracking of the feature projected into the image plane. The conversion from image-feature coordinates to mouse-pointer screen coordinates is typically implemented as a linear transformation in single-camera mouse-replacement systems. This means that the user is required to exert more efforts to move the mouse pointer in the outer regions of the computer screen than to move it within the center region of the screen. This may be particularly significant for individuals who have very limited head movements. It motivates implementations of nonlinear transfer functions [6], for example, based on distance to the center of the screen.

\section{Discussion and Conclusions}

We presented our research efforts towards developing a multi-camera mouse-replacement system for computer users with severe motion impairments. We have several years of experience working with individuals who use the "Camera Mouse," a publiclyavailable interface system that tracks the computer user's head movements with a single video camera and translates them into the movements of the mouse pointer on the computer screen. To address the problem that the Camera Mouse can lose track of facial features due to occlusion or spastic movements, we started to develop a multi-camera interface that provides (1) redundant input so that there is not a single point of tracking failure and (2) additional stereoscopic information to improve system reliability.

Our current multi-camera capture system can record synchronized images from multiple cameras showing different but, as typically desired, overlapping views of the same scene. Our system also automatically analyzes the geometry of the camera arrangement. It uses inexpensive webcams that can be placed on a desk in a typical human-computer interaction arrangement.

We used a three-camera version of our system to record 15 subjects while they were conducting a hands-free human-computer interaction experiment in real time. For this experiment, we developed a testing program that guided the subjects in making various head movements that resulted in significant mouse pointer movements and changes in movement directions. For each subject, we recorded three image sequences that were synchronized so that corresponding images were identifiable.

Our system provided the information about the geometry of any pair of cameras relative to one another. We reconstructed via stereoscopy the three-dimensional movement trajectories of various features such as the eyes and nose tip. Our analysis shows that single-camera interfaces based on two-dimensional feature tracking neglect to take into account the substantial feature movement in the third dimension. 

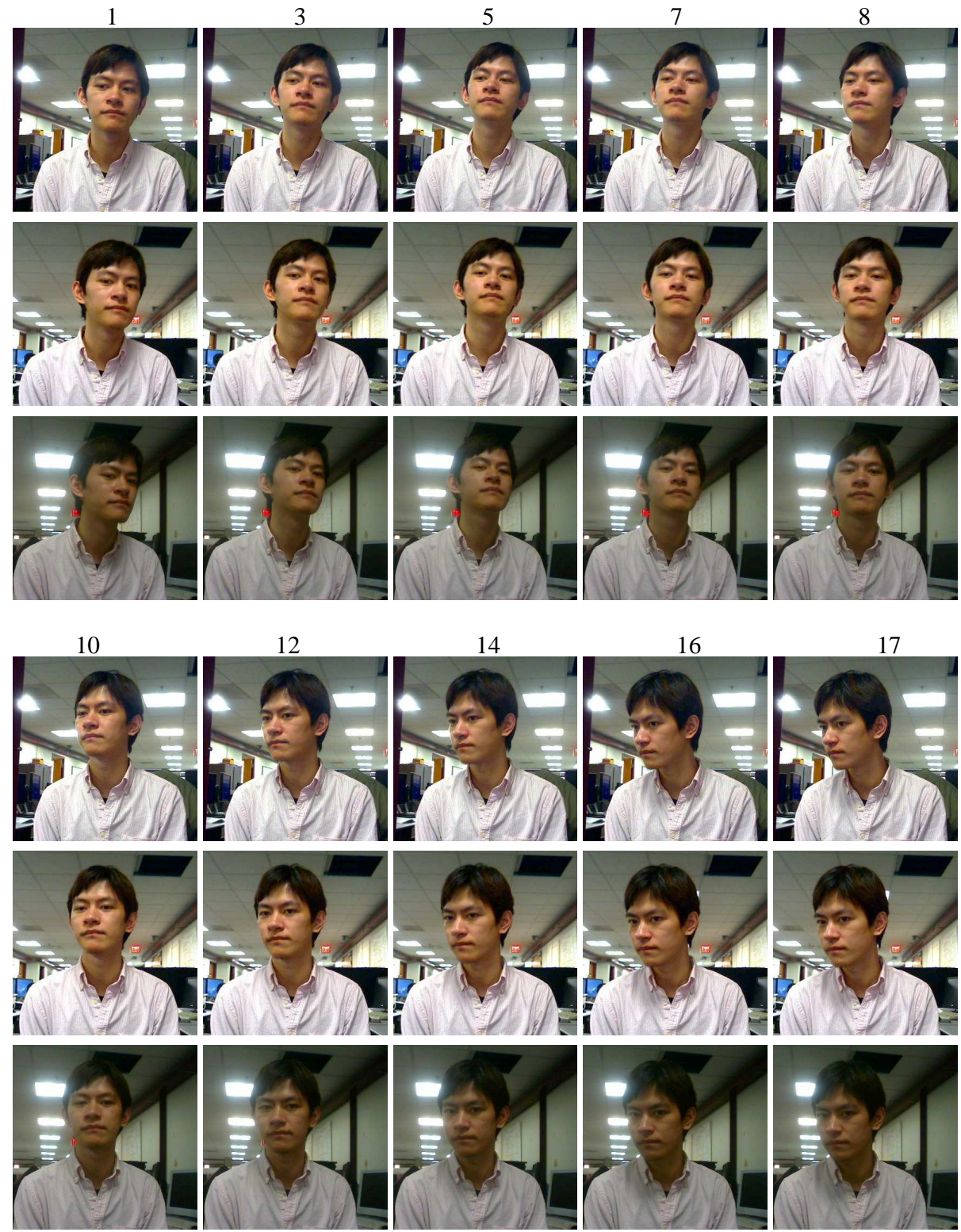

Fig. 5. Cropped images of a user during our human-computer interaction experiment with the ClickTester program. Each column shows simultaneously recorded images from the left, center, and right cameras with the corresponding frame number (time stamp) on top. During the experiment, the user first selected a target circle at the top of the screen, which resulted in a raising of his head. He then moved the mouse pointer to a target circle near the lower left corner of the screen, which resulted in a turning and lowering of his head. 

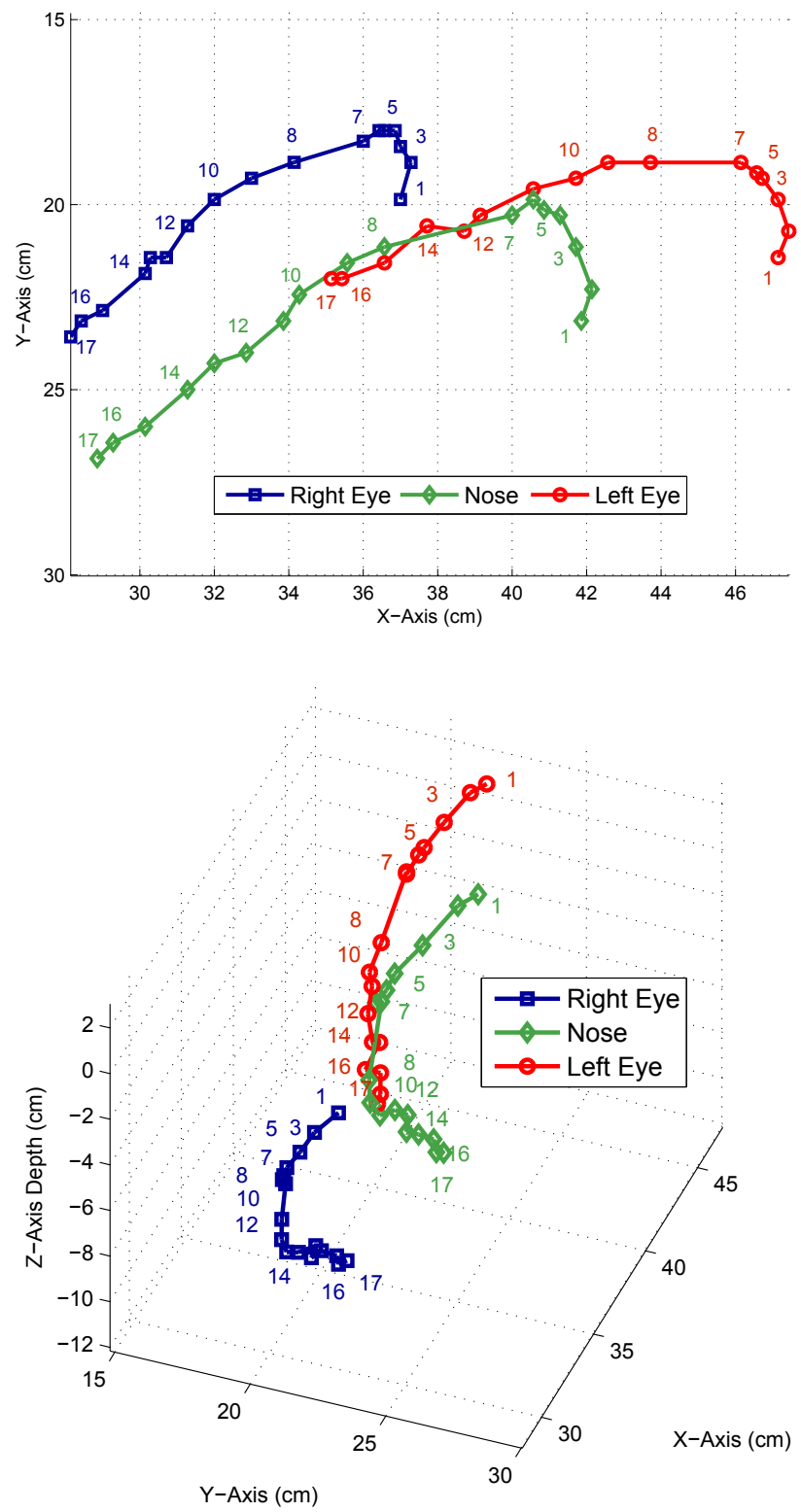

Fig. 6. Reconstructed 3D feature trajectories. Numbers indicate time stamps. Top: 3D points in the $x-y$ plane that is parallel to the left camera. Bottom: This viewing angle shows the significant feature movement in the $z$ direction, first away and then towards the camera.

\section{Acknowledgements}

We would like to thank the human subjects who spared time from their busy schedules to participate in the experiments. Funding for this work was provided by the National Science Foundation, HCC grant IIS-0713229. 


\section{References}

1. A. H. Gee and R. Cipolla. Tracking faces. In R. Cipolla and A. Pentland, editors, Computer Vision for Human-Machine Interaction, pages 113-122. Cambridge University Press, 1998.

2. W. T. Freeman, D. Anderson, P. Beardsley, C. Dodge, H. Kage, K. Kyuma, Y. Miyake, M. Roth, K. Tanaka, C. Weissman, and W. Yerazunis. Computer vision for interactive computer graphics. IEEE Computer Graphics and Applications, 18(3):42-53, May 1998.

3. M. Turk and R. George. Perceptual user interfaces. Comm. of the ACM, 43(3), 2000.

4. M. Betke, J. Gips, and P. Fleming. The camera mouse: Visual tracking of body features to provide computer access for people with severe disabilities. IEEE Transactions on Neural Systems and Rehabilitation Engineering, 10(1):1-10, 2002.

5. J. Varona, C. Manresa-Yee, and F. J. Perales. Hands-free vision-based interface for computer accessibility. Journal of Network and Computer Applications, 31(4):357-374, 2008.

6. R. Kjeldsen. Improvements in vision-based pointer control. In 8th International ACM SIGACCESS Conference on Computers and accessibility (Assets '06), pages 189-196, 2006.

7. D. O. Gorodnichy and G. Roth. Nouse 'use your nose as a mouse' perceptual vision technology for hands-free games and interfaces. Image and Vision Computing, 22(12):931-942, 2004.

8. E. A. Cansizoglu and M. Betke. An information fusion approach for multiview feature tracking. In 20th International Conference on Pattern Recognition (ICPR 2010), Istanbul, Turkey, 2010. 4 pp.

9. T. K. Marks, J. R. Hershey, and J. R. Movellan. Tracking motion, deformation, and texture using conditionally Gaussian processes. IEEE Transactions on Pattern Analysis and Machine Intelligence, 32(2):348-363, 2010.

10. C. John, U. Schwanecke, and H. Regenbrecht. Real-time volumetric reconstruction and tracking of hands and face as a user interface for virtual environments. In VR 2009: IEEE Virtual Reality Conference, pages 241-242, 2009.

11. M. Rätsch, C. Blumer, G. Teschke, and T. Vetter. Coarse-to-fine particle filters for multiobject human computer interaction. In IEEE International Workshop on Intelligent Data Acquisition and Advanced Computing Systems: Technology and Applications (IDAACS 2009), pages 440-445, September 2009.

12. K. Sidorov, Y. Hicks, D. Marshall, S. Sanei, and J. Chambers. Real time multi camera 3D tracking system. In 3rd European Conference on Visual Media Production (CVMP 2006), page 191, London, UK, 2006.

13. F. Dornaika and B. Raducanu. Three-dimensional face pose detection and tracking using monocular videos: Tool and application. IEEE Transactions on Systems, Man, and Cybernetics, Part B: Cybernetics, 39(4):935-944, 2009.

14. G. Bradski and A. Kaehler. Learning OpenCV, chapter 11-12. O'Reilly, 2008.

15. R. I. Hartley and A. Zisserman. Multiple View Geometry in Computer Vision. Cambridge University Press, 2003.

16. M. A. Fischler and R. C. Bolles. Random sample consensus: a paradigm for model fitting with applications to image analysis and automated cartography. Communications of the ACM, 24(6):381-395, 1981.

17. Camera Mouse - Innovative software for people with disabilities, http://www.cameramouse.org, accessed April 2010. 